\title{
Equivalent Relations Between Interchannel Coupling and Antenna Polarization Coupling in Polarization Diversity Systems
}

\author{
Xin Li, Xiujiang Huang, Zaiping Nie, Senior Member, IEEE, and Yimin Zhang, Senior Member, IEEE
}

\begin{abstract}
Both the interchannel coupling (CC) among multiple compact circuitry channels and the antenna polarization coupling (APC) due to angular deviation of antennas (ADA) in diversity systems cannot be neglected in practice. The effects of these two problems are considered in this paper. The investigation of the $\mathrm{CC}$ in a two-branch orthogonal polarization antenna diversity system is extended to the case of a general multibranch polarization antenna diversity system, and the $\mathrm{CC}$ is investigated together with APC by applying a network theory framework. Their effects on both the correlation and the mean power ratio among received signals are obtained. It is found that the effects of both kinds of couplings as well as that of the combining signal processing on the received signals can be considered as a series of weightings. Two equivalent relations among APC, CC, and ADA are developed. Such equivalences reveal insights into $\mathrm{CC}$ from the point of view of antennas and provide additional degree-of-freedom for system optimization to improve the diversity performance. Furthermore, two kinds of compensation techniques are also developed to mitigate the APC and $\mathrm{CC}$, as well as to enhance the diversity performance. Finally, some numerical results are presented to validate the analyses.
\end{abstract}

Index Terms-Angular deviation of antenna (ADA), antenna polarization coupling (APC), diversity, equivalent relations, interchannel coupling (CC).

\section{INTRODUCTION}

A NTENNA diversity is effective to combat fading in mobile wireless communications [1]. Since polarization diversity is nearly as effective as spatial diversity [2], the polarization antenna diversity scheme is commonly used because it can save space and compensate for polarization mismatch [3]. Both the correlation coefficients and the mean power ratio between two-branch signals have been discussed in order to determine diversity performance [4], [5], where a kind of coupling,

Manuscript received November 30, 2005; revised February 1, 2007. This work was supported in part by the National High-tech Research and Development Program of China under Contract 2002AA123032 and in part by the ONR/NSWC under Contract N65540-05-C-0028.

$\mathrm{X}$. Li was with the School of Electrical Engineering, University of Electronic Science and Technology of China, Chengdu, China. He is with the Center for Advanced Communications, Villanova University, Villanova, PA 19085 USA (e-mail: xin.1@villanova.edu).

X. Huang is with No. 23 Research Institute of China Electronics Technology Group Corporation, Shanghai 200437, China (e-mail: huangxj77@163.com).

Z. Nie is with the School of Electrical Engineering, University of Electronic Science and Technology of China, Chengdu 610054, China (e-mail: zpnie@uestc.edu.cn).

Y. Zhang is with the Center for Advanced Communications, Villanova University, Villanova, PA 19085 USA (e-mail: yimin.zhang@villanova.edu).

Digital Object Identifier 10.1109/TAP.2007.898508 namely antenna polarization coupling (APC), between two orthogonal polarization diversity antennas has also been studied. However, another kind of coupling, namely interchannel coupling (CC), among multiple parallel circuitry channels in diversity systems has attracted little research interest [12]-[14], although the impacts of mutual coupling among antenna elements have been investigated extensively [6]-[11]. Nevertheless, the CC among compact analog circuit blocks in multichannel systems cannot be neglected in practical situations, since numerous similar RF and IF circuitry channels are parallely integrated to reduce size and cost (e.g., in multiple-input multiple-output (MIMO) wireless systems, see [7], [9]-[11], and the references therein), yielding insufficient isolation among circuitry channels. Therefore, it is also necessary to analyze the $\mathrm{CC}$ in polarization antenna diversity systems [12], [13]. On the other hand, the angular deviation of antennas (ADA) in multiantenna systems cannot be neglected since physical implementation errors and installation deviations are inevitable [14]. Hence, the impact of ADA on the diversity performance has to be investigated.

In [12] and [13], we analyzed the effects of $C C$ in a twobranch orthogonal polarization antenna diversity system on both the correlation and the mean power ratio between the received signals. In this paper, the investigation of CC in a two-branch orthogonal polarization antenna diversity system is extended to the case of a general multibranch polarization antenna diversity system, and the CC is investigated together with APC by applying a network theory framework. It is found that the effects of both kinds of couplings as well as that of the processing in the combining signal processor (CSP) on the received signals can be considered as a series of weightings. As the result, two equivalent relations are developed. Such equivalent relations not only reveal insights into $\mathrm{CC}$ from the point of view of antennas, but also provide another degree-of-freedom for the optimization of the diversity performance. Further, two methods are proposed to compensate for the APC and CC, and the effectiveness and flexibility of these methods in improving the diversity performance are demonstrated.

The remainder of this paper is outlined as follows. Section II analyzes the APC and CC in two- and multibranch polarization antenna diversity systems by applying a network theory framework, and their effects on both the correlation and the mean power ratio among received signals are analyzed. Two equivalent relations among APC, CC and ADA are proposed in Section III, and some typical values of the relations are also provided. In Section IV, two kinds of compensation methods for APC and CC are proposed. Section V presents some numerical results to validate the previous analyses. Finally, we conclude this paper in Section VI. 


\section{ANALYSIS OF APC AND CC}

For a two-branch orthogonal polarization antenna system, the received signal at each polarized antenna can be independent of each other. A typical two-branch orthogonal polarization antenna diversity system is shown in Fig. 1, where horizontally polarized antenna $V_{1}$ and vertically polarized antenna $V_{2}$ are orthogonally co-placed. The polarized signals incident at the corresponding antennas can be represented as a $2 \times 1$ vector $\mathbf{r}_{\text {in }}=\left[r_{\text {in } 1}, r_{\text {in } 2}\right]^{\mathrm{T}}$, where superscript $\mathrm{T}$ denotes vector transpose. The elements of $\mathbf{r}_{\mathrm{in}}$ are expressed as

$$
r_{\text {in } j}=A_{j} \cos \left(\varphi_{j}\right), \quad j=1,2
$$

where $A_{j}$ and $\varphi_{j}$ are, respectively, the signal amplitude and phase of the $j$ th branch. In a typical multipath flat-fading environment, $A_{1}$ and $A_{2}$ have independent Rayleigh distributions, and $\varphi_{1}$ and $\varphi_{2}$ have independent uniform distributions [4], [5]. Under the assumption that there is no APC between antenna branches $\mathrm{V}_{1}$ and $\mathrm{V}_{2}$, both the correlation coefficient and the mean power ratio between two-branch signals were derived in [4], [5]. These results can be straightforwardly extended to general multibranch polarization antenna diversity systems. However, for an $N$-branch polarization antenna diversity system, there may exist APC among polarization orientations, i.e., the incident signals at the polarized antennas may be dependent to each other. In this case, by applying a network theory framework through the introduction of an $N \times N$ APC matrix $\mathbf{C}_{\text {ant }}$, the received signals at the corresponding antenna ports can be modified as

$$
\mathbf{r}_{\mathrm{out}}^{\mathrm{ant}}=\mathbf{C}_{\mathrm{ant}} \mathbf{r}_{\mathrm{in}} \text {. }
$$

In the absence of APC, the matrix of $\mathbf{C}_{a n t}$ is an identity matrix. The received signals with APC pass sequentially through the corresponding RF block, IF block, and analog-to-digital converter (ADC) block, and then arrive in terms of $\mathbf{r}_{\text {in }}^{\text {com }}$ at the input ports of the CSP, where an $N \times 1$ combining vector $\mathbf{C}_{\text {com }}$ is applied and yields

$$
r_{\mathrm{out}}=\mathbf{C}_{\mathrm{com}} \mathbf{r}_{\mathrm{in}}^{\mathrm{com}}
$$

It is often assumed that the isolation between two circuitry channels is sufficiently high and the unintentional $\mathrm{CC}$ is thus negligible. In practice, however, it is not very easy to avoid the $\mathrm{CC}$ in compact circuit designs. Therefore, it is necessary to take the $\mathrm{CC}$ into account and analyze its effects on the diversity performance of multichannel systems.

In the presence of unintentional $\mathrm{CC}$, we denote the $\mathrm{CC}$ coefficient $c_{\mathrm{ch} k j}$ to quantify the coupling from circuitry channel $j$ to $k$, and the transmission coefficient $c_{\operatorname{ch} j j}$ to quantify the transmission of channel $j$ itself. Consequently, the effects of $\mathrm{CC}$ can be taken into account by introducing an $N \times N \mathrm{CC}$ matrix $\mathbf{C}_{\mathrm{ch}}$. Then the input signals to the CSP become

$$
\mathbf{r}_{\mathrm{in}}^{\mathrm{com}}=\mathbf{C}_{\mathrm{ch}} \mathbf{r}_{\mathrm{out}}^{\text {ant }}
$$

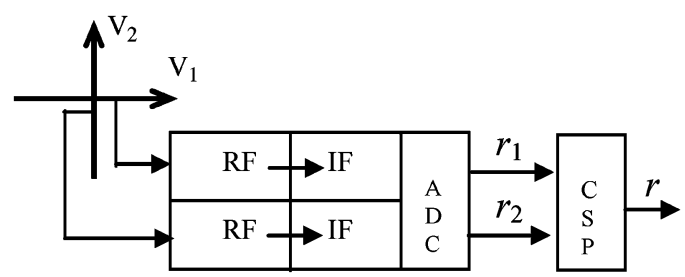

Fig. 1. Block diagram of the two-branch orthogonal polarization antenna diversity system.

In the absence of the unintentional $\mathrm{CC}$, the $\mathrm{CC}$ matrix $\mathrm{C}_{\mathrm{ch}}$ becomes an identity matrix.

When both kinds of couplings are considered, by inserting (2) into (4), one can get a more concise form of the received signals entering the CSP as

$$
\mathbf{r}_{\mathrm{in}}^{\mathrm{com}}=\mathbf{C}_{\mathrm{ch}} \mathbf{C}_{\mathrm{ant}} \mathbf{r}_{\mathrm{in}} \text {. }
$$

Furthermore, if the two kinds of couplings are considered concurrently, they can be incorporated into one combined coupling matrix $\mathbf{C}_{\mathrm{ch}-\text { ant }}$, which is the concatenation of two coupling matrices $\mathbf{C}_{\mathrm{ch}}$ and $\mathbf{C}_{\text {ant }}$

$$
\mathbf{r}_{\mathrm{in}}^{\mathrm{com}}=\mathbf{C}_{\mathrm{ch}-\mathrm{ant}} \mathbf{r}_{\mathrm{in}}, \quad \mathbf{C}_{\mathrm{ch}-\mathrm{ant}}=\mathbf{C}_{\mathrm{ch}} \mathbf{C}_{\mathrm{ant}} .
$$

Consequently, by inserting (6) into (3), the signal output $r_{\text {out }}$ of the CSP in such a multibranch polarization antenna diversity system can be obtained as

$$
r_{\mathrm{out}}=\mathbf{C}_{\mathrm{com}} \mathbf{C}_{\mathrm{ch}} \mathbf{C}_{\mathrm{ant}} \mathbf{r}_{\mathrm{in}}=\mathbf{C}_{\mathrm{com}} \mathbf{C}_{\mathrm{ch}-\mathrm{ant}} \mathbf{r}_{\mathrm{in}} .
$$

The analysis above shows that, if either APC or CC is considered, the received signals are subject to a weighting before they are fed to the CSP. If both kinds of couplings are taken into account, the received signals are equivalently subject to two concatenated weightings before entering the CSP. Finally, for a multibranch polarization antenna diversity system, the effects of both kinds of couplings, namely APC and CC, as well as the processing in the CSP on the received signals can be considered as a series of weightings.

As an example, we consider a two-branch orthogonal polarization antenna diversity system as shown in Fig. 1. Assume that no APC exists, i.e., the matrix of $\mathbf{C}_{\text {ant }}$ is the $2 \times 2$ identity matrix, and only the $\mathrm{CC}$ needs to be considered. Hence, the input signals to the CSP can be written as

$$
\begin{aligned}
& r_{\mathrm{in} 1}^{\mathrm{com}}=c_{\mathrm{ch} 11} r_{\mathrm{in} 1}+c_{\mathrm{ch} 12} r_{\mathrm{in} 2}, \\
& r_{\mathrm{in} 2}^{\mathrm{com}}=c_{\mathrm{ch} 21} r_{\mathrm{in} 1}+c_{\mathrm{ch} 22} r_{\mathrm{in} 2} .
\end{aligned}
$$

Intuitively, the correlation between the two received signals is increased due to the existence of $\mathrm{CC}$. Following the derivations of the correlation coefficient and the mean power ratio between two-branch signals [4], [5], the correlation between the two revised signals, $r_{\mathrm{in} 1}^{\mathrm{com}}$ and $r_{\mathrm{in} 2}^{\mathrm{com}}$, can be expressed as [12]-[14]

$$
\begin{aligned}
\rho_{r}=\langle & \left.\left(r_{\mathrm{in} 1}^{\mathrm{com}}-\left\langle r_{\mathrm{in} 1}^{\mathrm{com}}\right\rangle\right)\left(r_{\mathrm{in} 2}^{\mathrm{com}}-\left\langle r_{\mathrm{in} 2}^{\mathrm{com}}\right\rangle\right)^{*}\right\rangle \cdot \\
& \left(\left\langle\left|r_{\mathrm{in} 1}^{\mathrm{com}}-\left\langle r_{\mathrm{in} 1}^{\mathrm{com}}\right\rangle\right|^{2}\right\rangle\left\langle\left|r_{\mathrm{in} 2}^{\mathrm{com}}-\left\langle r_{\mathrm{in} 2}^{\mathrm{com}}\right\rangle\right|^{2}\right\rangle\right)^{-1 / 2}
\end{aligned}
$$


where $\langle\cdot\rangle$ expresses an ensemble average, and the asterisk denotes complex conjugate. By substituting (8) and (9) into (10), one can obtain

$$
\begin{aligned}
\rho_{r}= & \left(c_{\mathrm{ch} 11} c_{\mathrm{ch} 21}^{*}\left\langle\left|r_{\mathrm{in} 1}\right|^{2}\right\rangle+c_{\mathrm{ch} 12} c_{\mathrm{ch} 22}^{*}\left\langle\left|r_{\mathrm{in} 2}\right|^{2}\right\rangle\right) \\
& \cdot\left(\left|c_{\mathrm{ch} 11}\right|^{2}\left\langle\left|r_{\mathrm{in} 1}\right|^{2}\right\rangle+\left|c_{\mathrm{ch} 12}\right|^{2}\left\langle\left|r_{\mathrm{in} 2}\right|^{2}\right\rangle\right)^{-1 / 2} \\
& \cdot\left(\left|c_{\mathrm{ch} 21}\right|^{2}\left\langle\left|r_{\mathrm{in} 1}\right|^{2}\right\rangle+\left|c_{\mathrm{ch} 22}\right|^{2}\left\langle\left|r_{\mathrm{in} 2}\right|^{2}\right\rangle\right)^{-1 / 2} .
\end{aligned}
$$

Let the cross-polar discrimination (XPD) of two polarized signals be

$$
\Gamma=\left\langle\left|r_{\mathrm{in} 2}\right|^{2}\right\rangle /\left\langle\left|r_{\mathrm{in} 1}\right|^{2}\right\rangle .
$$

In a typical real environment, $\Gamma>1$ is usually satisfied, because the signal received at the vertical polarization antenna in channel 2 is often stronger than that received at the horizontal polarization antenna in channel 1 . Inserting (12) into (11), one obtains the analytical expression of the correlation with CC in a two-branch orthogonal polarization system, expressed as

$$
\begin{array}{r}
\rho_{r}=\left(c_{\mathrm{ch} 11} c_{\mathrm{ch} 21}^{*}+c_{\mathrm{ch} 12} c_{\mathrm{ch} 22}^{*} \Gamma\right) \cdot\left(\left|c_{\mathrm{ch} 11}\right|^{2}+\left|c_{\mathrm{ch} 12}\right|^{2} \Gamma\right)^{-1 / 2} \\
\cdot\left(\left|c_{\mathrm{ch} 21}\right|^{2}+\left|c_{\mathrm{ch} 22}\right|^{2} \Gamma\right)^{-1 / 2} \cdot
\end{array}
$$

It is clear from this result that the received signals become correlated in the presence of the $\mathrm{CC}$ between channels, and the correlation depends on the $\mathrm{CC}$ coefficients, the circuitry channel transmission coefficients as well as the XPD. Equation (13) also shows that the correlation increases with $\mathrm{CC}$ coefficients and, because $\Gamma>1, c_{\mathrm{ch} 12}$ has a more significant effect on the correlation than $c_{\mathrm{ch} 21}$ does.

In the presence of the $\mathrm{CC}$ between channels, the mean received power ratio is given by

$$
L=\left\langle\left|r_{\text {in2 }}^{\text {com }}\right|^{2}\right\rangle /\left\langle\left|r_{\text {in1 }}^{\text {com }}\right|^{2}\right\rangle .
$$

Substituting (8), (9), and (12) into (14), one obtains

$$
L=\left(\left|c_{\mathrm{ch} 21}\right|^{2}+\left|c_{\mathrm{ch} 22}\right|^{2} \Gamma\right) /\left(\left|c_{\mathrm{ch} 11}\right|^{2}+\left|c_{\mathrm{ch} 12}\right|^{2} \Gamma\right)
$$

which shows that the $\mathrm{CC}$ between the two channels affects the mean received power ratio and, hence, the XPD. Equation (15) also shows that the CC can either decrease or increase $L$, but the former is more probable in a real environment where $\Gamma>1$ holds.

\section{EQuivalent Relations BetweEn APC AND CC}

The analyses in Section II have shown that, for a multibranch polarization antenna diversity system, both APC and CC can be represented as proper weightings and, if both of them are taken into account, the received signals are equivalently subject to two concatenated weightings before entering the CSP. These results explicitly indicate that the two kinds of couplings are equivalent in the sense of their impacts on the polarized signals incident at the antennas. Hence, similar to CC, APC also not only increases the correlation but also affects the mean power ratio between two received signals.

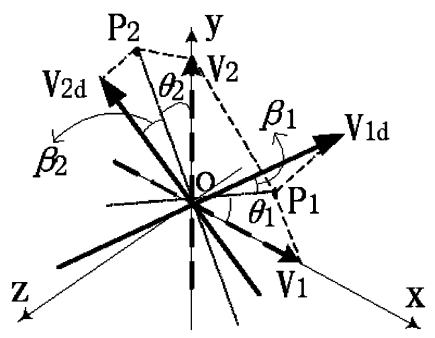

Fig. 2. Geometrical diagram of the deviation angles of a two-branch orthogonal polarization antenna.

For a widely used orthogonal polarization antenna diversity system, this equivalence becomes very useful. For example, for a typical two-branch orthogonal polarization antenna system, as shown in Fig. 1, the received signals at the polarized antennas are mutually independent and, hence, in theory, there is no APC between antenna $V_{1}$ and $V_{2}$. However, in practice, there exists an unintentional ADA between $V_{1}$ and $V_{2}$, which results from, for example, physical implementation errors and installation deviations as shown in Fig. 2, where it is assumed that antenna $V_{1}$ is rotated around the origin to $\mathrm{V}_{1 \mathrm{~d}}$, which deviates an angle of $\beta_{1}$ from the $x-y$ plane, and its projection $\mathrm{OP}_{1}$ to the $x-y$ plane makes an angle of $\theta_{1}$ with $x$ axis. Similarly, antenna $V_{2}$ is rotated around the origin to $\mathrm{V}_{2 \mathrm{~d}}$, with an angle $\beta_{2}$ from the $x-y$ plane and $\theta_{2}$ of its projection $\mathrm{OP}_{2}$ to the $x-y$ plane with $y$ axis. The received signals of the antenna $V_{1 d}$ and $V_{2 d}$ entering the CSP in the absence of CC can be written as

$$
\begin{aligned}
& r_{\text {in1d }}^{\text {com }}=r_{\text {in } 1} \cos \theta_{1} \cos \beta_{1}+r_{\text {in } 2} \sin \theta_{1} \cos \beta_{1} \\
& r_{\text {in2d }}^{\text {com }}=-r_{\text {in } 1} \sin \theta_{2} \cos \beta_{2}+r_{\text {in } 2} \cos \theta_{2} \cos \beta_{2}
\end{aligned}
$$

which show that the ADA in orthogonally polarized antenna diversity systems results in the APC. Since the APC and CC have equivalent impacts on the polarized signals incident at the antennas, the CC and ADA also have equivalent impacts in the same sense. Comparing (8), (9), (16), and (17), it is evident that the angular deviations of orthogonally polarized antennas and the $\mathrm{CC}$ are equivalent in the sense of their impacts on the polarized signals incident at the antennas. In this case, either of the following two groups of equations has to be satisfied

$$
\left\{\begin{array} { l } 
{ r _ { \text { in1 } } ^ { \text { com } } = r _ { \text { in1d } } ^ { \text { com } } } \\
{ r _ { \text { in2 } } ^ { \text { com } } = r _ { \text { in2d } } ^ { \text { com } } }
\end{array} \quad \text { (i) or } \quad \left\{\begin{array}{l}
r_{\text {in1 }}^{\text {com }}=r_{\text {in2d }}^{\text {com }} \\
r_{\text {in2 }}^{\text {com }}=r_{\text {in1d }}^{\text {com }}
\end{array} \quad\right.\right. \text { (ii). }
$$

In the case of (18-i), one obtains

$$
\begin{cases}c_{\mathrm{ch} 11}=\cos \theta_{1} \cos \beta_{1} ; & c_{\mathrm{ch} 12}=\sin \theta_{1} \cos \beta_{1} \\ c_{\mathrm{ch} 21}=-\sin \theta_{2} \cos \beta_{2} ; & c_{\mathrm{ch} 22}=\cos \theta_{2} \cos \beta_{2} .\end{cases}
$$

In the case of (18-ii), one obtains

$$
\begin{cases}c_{\mathrm{ch} 11}=-\sin \theta_{2} \cos \beta_{2} ; & c_{\mathrm{ch} 12}=\cos \theta_{2} \cos \beta_{2} \\ c_{\mathrm{ch} 21}=\cos \theta_{1} \cos \beta_{1} ; & c_{\mathrm{ch} 22}=\sin \theta_{1} \cos \beta_{1} .\end{cases}
$$

Both (19) and (20) show that the ADA is equivalent to the CC in two-branch polarization antenna diversity systems, and the equivalent $\mathrm{CC}$ and transmission coefficients are determined by either (19) or (20). If all the deviation angles are real, the CC and transmission coefficients are also real. Furthermore, only (19) is satisfied under the constraints that the CC coefficients are zeros and the transmission coefficients are ones in the absence of the 
TABLE I

TyPICAL VALUES OF THE EQUIVALENT RELATION BETWEEN THE ADA (UNITS: DEGREES) AND CC, BASED ON (19)

\begin{tabular}{cccc|cccc}
\hline$\theta_{1}$ & $\theta_{2}$ & $\beta_{2}$ & $\beta_{2}$ & $c_{\text {ch11 }}$ & $c_{\text {ch21 }}$ & $c_{\text {ch12 }}$ & $C_{\text {ch22 }}$ \\
\hline 0 & 0 & 0 & 0 & 1 & 0 & 0 & 1 \\
1 & 1 & 0 & 0 & 0.9998 & -0.0175 & 0.0175 & 0.9998 \\
0 & 0 & 1 & 1 & 0.9998 & 0 & 0 & 0.9998 \\
1 & 1 & 1 & 1 & 0.9997 & -0.0174 & 0.0174 & 0.9997 \\
5 & 5 & 5 & 5 & 0.9924 & -0.0868 & 0.0868 & 0.9924 \\
10 & 10 & 10 & 10 & 0.9698 & -0.1710 & 0.1710 & 0.9698 \\
20 & 20 & 20 & 20 & 0.8830 & -0.3214 & 0.3214 & 0.8830 \\
30 & 30 & 30 & 30 & 0.7500 & -0.4330 & 0.4330 & 0.7500 \\
30 & 30 & 0 & 0 & 0.8660 & -0.5000 & 0.5000 & 0.8660 \\
45 & 45 & 0 & 0 & 0.7071 & -0.7071 & 0.7071 & 0.7071 \\
45 & 45 & 45 & 45 & 0.5000 & -0.5000 & 0.5000 & 0.5000 \\
\hline
\end{tabular}

ADA. As an example, Table I provides some typical values of the equivalent relation between the ADA and CC based on (19).

Conversely, (19) and (20) also indicate that the $\mathrm{CC}$ is equivalent to the ADA in two-branch polarization diversity systems, and the equivalent deviation angles are also determined by either (19) or (20). In the case of (19), one obtains

$\begin{cases}\theta_{1}=\operatorname{atan}\left(c_{\mathrm{ch} 12} / c_{\mathrm{ch} 11}\right) ; & \theta_{2}=-\operatorname{atan}\left(c_{\mathrm{ch} 21} / c_{\mathrm{ch} 22}\right) \\ \beta_{1}= \pm \operatorname{acos}\left(\sqrt{c_{\mathrm{ch} 11}^{2}+c_{\mathrm{ch} 12}^{2}}\right) ; & \beta_{2}= \pm \operatorname{acos}\left(\sqrt{c_{\mathrm{ch} 21}^{2}+c_{\mathrm{ch} 22}^{2}}\right) .\end{cases}$

In the case of (20), one obtains

$$
\begin{cases}\theta_{1}=\operatorname{atan}\left(c_{\mathrm{ch} 22} / c_{\mathrm{ch} 21}\right) ; & \theta_{2}=-\operatorname{atan}\left(c_{\mathrm{ch} 11} / c_{\mathrm{ch} 12}\right) \\ \beta_{1}= \pm \operatorname{acos}\left(\sqrt{c_{\mathrm{ch} 21}^{2}+c_{\mathrm{ch} 22}^{2}}\right) ; & \beta_{2}= \pm \operatorname{acos}\left(\sqrt{c_{\mathrm{ch} 11}^{2}+c_{\mathrm{ch} 12}^{2}}\right) .\end{cases}
$$

These results show that, if the $\mathrm{CC}$ and transmission coefficients are real, four equivalent ADA parameters are also real. However, if the $\mathrm{CC}$ and transmission coefficients are complex, some of the equivalent ADA parameters might also be complex. A complex ADA coefficient implies a real amplitude weighting and a phase shift for the polarized signal incident at the antenna. To explain this, we express $\theta_{1}$ as $\theta_{1}=\theta_{1 x}+i \theta_{1 y}$, where $\theta_{1 x}$ and $\theta_{1 y}$ are real, and $i=\sqrt{-1}$. Then, $\sin \left(\theta_{1}\right)$ can be expressed exponentially as

$$
\begin{aligned}
\sin \theta_{1} & =a_{\theta_{1}} e^{i \theta_{1}^{\text {new }}} \\
a_{\theta_{1}} & =\left[\left(\sin \theta_{1 x} \cosh \theta_{1 y}\right)^{2}+\left(\cos \theta_{1 x} \sinh \theta_{1 y}\right)^{2}\right]^{1 / 2} \\
\theta_{1}^{\text {new }} & =\operatorname{atan}\left(\cos \theta_{1 x} \sinh \theta_{1 y} / \sin \theta_{1 x} \cosh \theta_{1 y}\right) .
\end{aligned}
$$

Thus, it becomes clear that $a_{\theta_{1}}$ is a real amplitude weighting and $\theta_{1}^{\text {new }}$ is a phase shift for the polarized signal incident at the antenna. That is, a complex deviation angle for the antenna orientation is same as a complex weighting for the polarized signal incident at the antenna. Furthermore, the real amplitude weighting in (23) can be written as

$$
\begin{aligned}
a_{\theta_{1}} & =\sin \theta_{1}^{a} \\
\theta_{1}^{a} & =\operatorname{asin}\left\{\left[\left(\sin \theta_{1 x} \cosh \theta_{1 y}\right)^{2}+\left(\cos \theta_{1 x} \sinh \theta_{1 y}\right)^{2}\right]^{1 / 2}\right\} .
\end{aligned}
$$

Inserting (24) into (23), it is clear that a complex deviation angle for the antenna orientation is also equivalent to a real deviation angle for the antenna orientation and a phase shift for the polarized signal incident at the antenna.
Additionally, according to the equivalent relations between the ADA and CC and by substituting (19) into (13) and (15), respectively, one promptly obtains both the correlation and the mean power ratio between the received signals of antennas $V_{1 d}$ and $\mathrm{V}_{2 \mathrm{~d}}$ in the absence of $\mathrm{CC}$ but in the presence of ADA as

$$
\begin{aligned}
\rho_{r}= & \left(\cos \beta_{1} \cos ^{*} \beta_{2}\right)\left|\cos \beta_{1} \cos \beta_{2}\right|^{-1} \\
& \cdot\left(\left|\cos \theta_{1}\right|^{2}+\left|\sin \theta_{1}\right|^{2} \Gamma\right)^{-1 / 2} \\
& \cdot\left(\left|\sin \theta_{2}\right|^{2}+\left|\cos \theta_{2}\right|^{2} \Gamma\right)^{-1 / 2} \\
& \cdot\left(\Gamma \sin \theta_{1} \cos ^{*} \theta_{2}-\cos \theta_{1} \sin ^{*} \theta_{2}\right) \\
L= & \left|\cos \beta_{2} / \cos \beta_{1}\right|^{2} \cdot\left(\left|\sin \theta_{2}\right|^{2}+\left|\cos \theta_{2}\right|^{2} \Gamma\right) \\
& \cdot\left(\left|\cos \theta_{1}\right|^{2}+\left|\sin \theta_{1}\right|^{2} \Gamma\right)^{-1}
\end{aligned}
$$

respectively. These results show that both the correlation and the mean power ratio are affected by the four ADA parameters. In effect, the impact of $\mathrm{CC}$ on the performance of diversity systems is equivalent to that of ADA, and vice versa. In particular, as mentioned above, if the $\mathrm{CC}$ and transmission coefficients are both real, the equivalent deviation angles are also real, and then the correlation is independent of the deviation angles from the vertical plane, i.e., $\beta_{1}$ and $\beta_{2}$. Furthermore, if the two deviation angles from the vertical plane are equal, both the correlation and the mean power ratio are independent of them, since both of them have converse contributions to the mean received power of two antennas.

In summary, the equivalent relations between the $\mathrm{CC}$ and APC not only reveal insights into $\mathrm{CC}$ from the point of view of antennas, but also provide another degree-of-freedom for the optimization of the diversity system to improve the performance. Such equivalences also bridge the $\mathrm{CC}$ and $\mathrm{ADA}$ in polarization antenna diversity systems and result in easy understanding of the equivalent relationships between them.

\section{COMPENSATION OF CC AND APC}

The equivalences between the $\mathrm{CC}$ and $\mathrm{APC}$, as mentioned in Section III, make it possible to achieve compensation of CC and APC. Such equivalences provide us an idea that, if either coupling is detrimental to diversity performance, it is possible to effectively reduce such detrimental impact by properly adjusting the other coupling. Furthermore, the equivalences provide another degree-of-freedom for the system optimization by adjusting one or both of the couplings in multibranch polarization antenna diversity systems. For example, in orthogonal polarization antenna diversity systems, we can make the combined coupling matrix $\mathbf{C}_{\mathrm{ch}-\text { ant }}$ in (6) approximately equal to the identity matrix by rotating the corresponding polarized antennas, i.e.

$$
\mathbf{C}_{\mathrm{ch}-\text { ant }}=\mathbf{C}_{\mathrm{ch}} \mathbf{C}_{\mathrm{ant}} \approx \mathbf{I} \text {. }
$$

Thus, the detrimental impact resulted from either the CC or APC can be reduced or compensated for by adjusting the other one.

Of course, there exist situations where approximating the combined coupling matrix $\mathbf{C}_{\mathrm{ch}-\text { ant }}$ to the identity matrix is difficult. In this case, another method can be applied. Before combining signal processing in the CSP, intentional interchannel coupling (ICC), denoted by an $N \times N$ matrix $\mathbf{C}_{\text {icc }}$, 


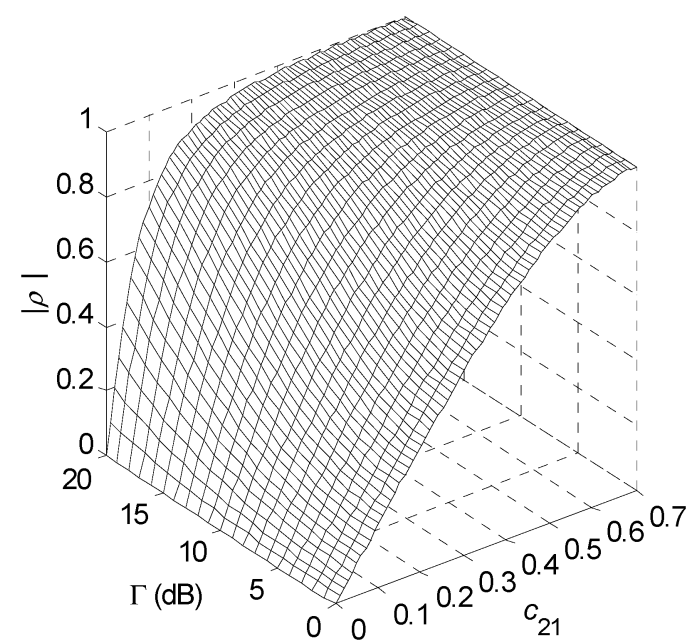

Fig. 3. Correlation between two received signals with $C C$ vs. both XPDs $(\Gamma)$ and $\mathrm{CC}$ coefficients $\left(c_{21}\right)$, assuming circuitry channels are symmetric and lossless.

can be introduced either by elaborating additional hardware circuits or by software processing in the CSP, such that

$$
\mathrm{C}_{\mathrm{icc}} \mathbf{C}_{\mathrm{ch}-\mathrm{ant}} \approx \mathbf{I} \text {. }
$$

Consequently, matrix $\mathbf{C}_{\text {icc }}$ can be determined as

$$
\mathbf{C}_{\mathrm{icc}} \approx \mathbf{C}_{\mathrm{ch}-\mathrm{ant}}^{-1}=\left(\mathbf{C}_{\mathrm{ch}} \mathbf{C}_{\mathrm{ant}}\right)^{-1}
$$

It shows that once the $\mathrm{CC}$ and $\mathrm{APC}$ matrices are available, the ICC matrix can be obtained to compensate for the CC and APC.

Additionally, besides the compensation of $\mathrm{CC}$ and $\mathrm{APC}$, it is possible to intentionally introduce more couplings $\mathbf{C}_{\text {icc-more }}$ among the received signals simply by adjusting the ICC matrix so as to improve the diversity performance. In this case, the ICC matrix can be expressed as

$$
\mathbf{C}_{\mathrm{icc}}=\mathbf{C}_{\mathrm{icc}-\mathrm{more}}\left(\mathbf{C}_{\mathrm{ch}} \mathbf{C}_{\mathrm{ant}}\right)^{-1} \text {. }
$$

Admittedly, a high efficiency of this method depends upon a low condition number of the combined coupling matrix $\mathbf{C}_{\mathrm{ch}-\mathrm{ant}}$, which means that the $\mathrm{CC}$ should be kept at a relatively low level.

\section{NUMERICAL ANALYSIS}

Assuming that the circuitry channels are symmetric and lossless, i.e., $c_{12}=c_{21},\left|c_{12}\right|^{2}+\left|c_{22}\right|^{2}=1$ and $\left|c_{11}\right|^{2}+\left|c_{21}\right|^{2}=1$ (For simplicity, the subscript 'ch' previously used in all CC coefficients is ignored hereafter). Numerical results of the correlation with CC between channels are illustrated in Fig. 3, and the mean power ratio $L$ is shown in Fig. 4 . It is observed in Fig. 3 that the correlation increases with either $\mathrm{CC}$ coefficients or XPDs. On the one hand, for a certain value of $\Gamma$, the correlation increases almost linearly with the CC coefficients as long as $\Gamma<6 \mathrm{~dB}$, and the increment becomes almost logarithmical

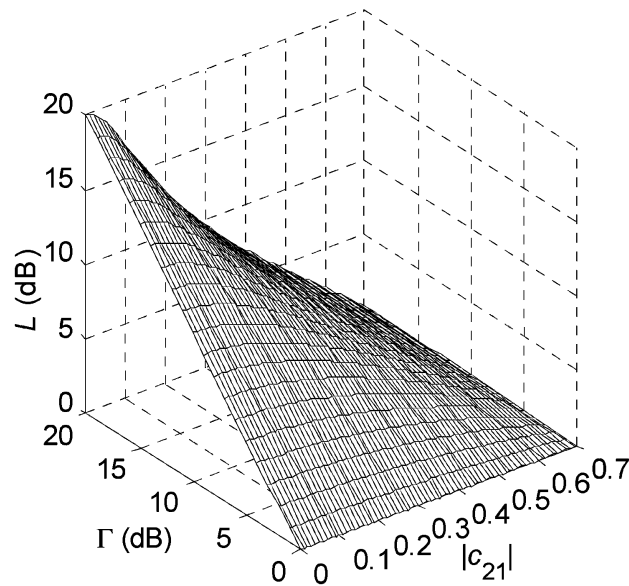

Fig. 4. The mean power difference between two received signals with $\mathrm{CC}$ vs. both XPDs $(\Gamma)$ and $\mathrm{CC}$ coefficients $\left(c_{21}\right)$, assuming channels are symmetric and lossless.

with the $\mathrm{CC}$ coefficients when $\Gamma>6 \mathrm{~dB}$. Therefore, reducing $\mathrm{CC}$ is efficient to decrease the correlation. On the other hand, for a certain $\mathrm{CC}$ coefficient, the correlation increases almost exponentially with $\Gamma$ if $\left|c_{21}\right|<0.15$, and for a higher $\left|c_{21}\right|$, the increment becomes almost linear with $\Gamma$ until it approaches the maximum. Fig. 4 shows that the mean power ratio decreases as $\left|c_{21}\right|$ increases. The value of $L$ slowly decreases almost linearly with $\left|c_{21}\right|$ for a low value of $\Gamma$ (i.e., $\Gamma<3 \mathrm{~dB}$ ), and the decrement becomes faster when $\Gamma$ is higher. For a low $C C$ coefficient, e.g., $\left|c_{12}\right|<0.1, L$ increases linearly with $\Gamma$, and the increment becomes slower when $\left|c_{21}\right|$ takes a larger value.

In general channels, it is possible that $c_{12} \neq c_{21}$. The correlation with CC coefficients was illustrated in [12] for $\Gamma=5 \mathrm{~dB}$ (see its Fig. 1), which shows that the correlation increases with the $\mathrm{CC}$ coefficients. The correlation approaches the maximum of 1 as the CC coefficients approach 0.7. It is noted that the impact of $c_{12}$ on the correlation is more significant than $c_{21}$, since the coupling results in stronger received signals in channel 2. Also, for general channels, the mean power ratio $L$ was provided in [12] for the case of $\Gamma=5 \mathrm{~dB}$ (see its Fig. 2), which indicates that the effect of $c_{12}$ on $L$ is more significant than $c_{21}$ due to the same reason. For a certain $c_{12}, L$ increases monotonically with $c_{21}$, and the increment goes faster as $c_{21}$ increases. This is due to the fact that the signal in the weaker channel is coupled into the stronger channel and results in a higher $L$.

It becomes clear from the above discussion that the CC increases the correlation coefficients but decreases the mean power difference, since the signal in a stronger channel is coupled into a weaker channel. Consequently, the CC has a contradictory impact on the diversity gain, since a high value of the diversity gain, which is a commonly used measure for the diversity performance, is obtained from a low correlation and a low mean power difference [4], [5]. It therefore creates a tradeoff between the optimistic and pessimistic impacts. The CC between channels is likely to become an optimistic factor for improving the diversity gain.

The diversity gain is also related to the method used for combining. The maximal ratio combining (MRC) is used here as an 


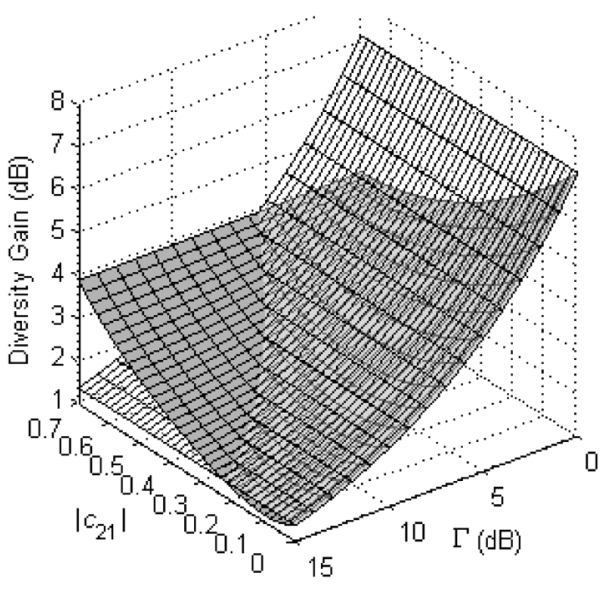

Fig. 5. Diversity gain of MRC for combining two received signals with $\mathrm{CC}$ (shadowed curve plane) and without CC (unshadowed curve plane) between symmetric and lossless channels.

example. Substituting both (11) and (13) in this paper into (5) in [2], which is an empirical relationship relating the diversity gain of MRC to both the mean power ratio and the correlation between signals, one obtains the diversity gain of MRC for combining two received signals with and without $\mathrm{CC}$ between general channels under a certain XPD (which was illustrated in [12], see its Fig. 3. It shows that the CC between general channels can either decrease or increase the diversity gain). Furthermore, the diversity gain of MRC for combining two received signals with and without CC in symmetric lossless channels is shown in Fig. 5, which shows that the $\mathrm{CC}$ between symmetric lossless channels can also affect the diversity gain, either constructively or destructively. When $\left|c_{21}\right|>0.2$ and $\Gamma>5 \mathrm{~dB}$, the diversity gain in the presence of $\mathrm{CC}$ (shadowed curve plane) is higher than that in the absence of CC (unshadowed curve plane). Under the conditions above, the $\mathrm{CC}$ becomes an optimistic factor for improving the diversity gain, which validates the aforementioned analyses. It is observed from other additional numerical results that the higher the $\Gamma$, the looser the conditions for the $\mathrm{CC}$ to improve the diversity gain. For example, if $\Gamma=10 \mathrm{~dB}$, the condition becomes loosened as $\left|c_{12}\right|>0.3$. In summary, a high value of XPD is necessary to improve the diversity gain in the presence of CC. This is due to the fact that it is by decreasing the mean power difference between the received polarized signals that the CC improves the diversity gain of a polarization antenna diversity system. Hence, if the mean power difference between the polarized incident signals at antenna ports is already very low, there is no much space left for the CC to improve the diversity gain.

\section{CONCLUSION}

The investigation of $\mathrm{CC}$ in a two-branch orthogonal polarization antenna diversity system has been extended to the case of a general multibranch polarization antenna diversity system, and the $\mathrm{CC}$ has been analyzed together with that of APC by applying a network theory framework in this paper. The effects of these two kinds of couplings on both the correlation and the mean power ratio among received signals were analyzed. It was found that the effects of both couplings and the processing in the CSP on the received signals can be considered as a series of weightings. Both the APC and CC increase the correlation but may decrease the mean power difference among the received signals. Hence, the APC and CC can be optimistic factors for improving the diversity performance if the mean power difference is pronounced. Such improvement is achieved by decreasing the mean power difference between the received polarized signals.

In addition, two equivalent relations among APC, CC and ADA were developed. These two kinds of couplings are equivalent in the sense that they have equivalent impacts on the polarized signals incident at the antennas. They are also equivalent in the same sense to the angular deviations of orthogonally polarized antennas. Such equivalent relations reveal insights into $\mathrm{CC}$ from the point of view of antennas, and provide additional degree-of-freedom for the optimization of the diversity systems and the improvement of the diversity gain performance.

Furthermore, two methods that compensate for the $\mathrm{CC}$ and APC were also proposed. It is possible to improve the diversity performance by adjusting one or both kinds of couplings, or by introducing intentional $\mathrm{CC}$ either through the elaboration of additional hardware circuits or software processing in the CSP. These methods are most effective when the combined coupling matrix has a relatively low condition number.

\section{REFERENCES}

[1] C. B. Dietrich, K. Dietze, J. R. Nealy, and W. L. Stutzman, "Spatial, polarization, and pattern diversity for wireless handheld terminals," IEEE Trans. Antennas Propag., vol. 49, no. 9, pp. 1271-1281, Sep. 2001.

[2] A. M. D. Turkmani, A. A. Arowojolu, P. A. Jefford, and C. J. Kellett, "An experimental evaluation of the performance of two-branch space and polarization diversity schemes at $1800 \mathrm{MHz}$," IEEE Trans. Veh. Technol., vol. 44, no. 2, pp. 318-326, May 1995.

[3] D. C. Cox, "Antenna diversity performance in mitigating the effects of portable radio telephone orientation and multipath propagation," IEEE Trans. Commun., vol. 31, no. 5, pp. 620-628, May 1983.

[4] S. Kozono, H. Tsuruhara, and M. Sakamoto, "Base station polarization diversity reception for mobile radio," IEEE Trans. Veh. Technol., vol. 33, no. 4, pp. 301-306, Nov. 1984.

[5] R. G. Vaughan, "Polarization diversity in mobile communications," IEEE Trans. Veh. Technol., vol. 39, no. 3, pp. 177-186, Aug. 1990.

[6] I. J. Gupta and A. K. Ksienski, "Effect of mutual coupling on the performance of adaptive arrays," IEEE Trans. Antennas Propag., vol. 31, no. 5, pp. 785-791, Sep. 1983.

[7] X. Li and Z. P. Nie, "Effect of mutual coupling on performance of MIMO wireless channels," in Proc 4th Int. Conf. Microw. Millimeter Wave Technol. (ICMMT), Beijing, China, Aug. 2004, pp. 150-153.

[8] R. Janaswamy, "Effect of element mutual coupling on the capacity of fixed length linear arrays," IEEE Antennas Wireless Propag. Lett., vol. 1, pp. 157-160, 2002.

[9] P. N. Fletcher, M. Dean, and A. R. Nix, "Mutual coupling in multielement array antennas and its influence on MIMO channel capacity," Electron. Lett., vol. 39, no. 4, pp. 342-344, Feb. 2003.

[10] X. Li and Z. P. Nie, "Effect of array orientation on performance of MIMO wireless channels," IEEE Antennas Wireless Propag. Lett., vol. 3, no. 16, pp. 368-371, Dec. 2004.

[11] X. Li and Z. P. Nie, "Mutual coupling effects on the performance of MIMO wireless channels," IEEE Antennas Wireless Propag. Lett., vol. 3, no. 16, pp. 344-347, Dec. 2004.

[12] X. Li and Z. P. Nie, "Channel coupling in orthogonal polarization diversity systems and its influence on diversity gain," Electron. Lett., vol. 41, no. 5, pp. 225-226, Mar. 2005.

[13] X. Li, Z. P. Nie, and X. J. Huang, "Channel coupling in orthogonal polarization diversity systems and its influence on diversity performances," in Proc. IEEE AP-S Int., Jul. 2005, vol. 1A, pp. 72-75.

[14] X. Li, Z. P. Nie, and X. J. Huang, "Equivalent relation between antennas angular deviations and channel coupling in orthogonal polarization diversity systems," in Proc. IEEE AP-S Int., Jul. 2005, vol. 1A, pp. 76-79. 


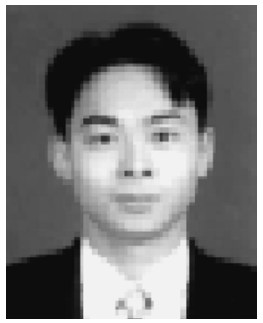

Xin Li was born in Sichuan, China, in 1975. He received the B.S. degree in electrical engineering and the M.S. degree in information and communications engineering from Chongqing University of Posts and Telecommunications (CQUPT), Chongqing, China, in 1999 and 2002, respectively. He received the $\mathrm{Ph} . \mathrm{D}$. degree in information and communications engineering and electromagnetic theory from University of Electronic Science and Technology of China (UESTC) in 2005.

From December 1999 to May 2001, he was a research engineer conducting work on his M.S. degree thesis with Datang Technology Co., Ltd., Beijing, where he also participated in drafting and amending the $3 \mathrm{G}$ time division synchronous code division multiple access (TD-SCDMA) standard, and in prototyping the TD-SCDMA/GSM system. From January to July 2002, he was a research engineer with Holley Co., Ltd., Dallas, TX, where he developed the dual-mode handset in cooperation with the R\&D Department. From July 2005 to August 2006, he was with Alcatel Shanghai Bell Research and Innovation Center as a Research Scientist, where he took part in the prototyping of MIMO-OFDM systems for future $4 \mathrm{G}$ systems. He is currently a Postdoctoral Research Fellow with the Center for Advanced Communications, Villanova University, Villanova, PA. His research interests include MIMO radio transmission technologies, OFDM, CDMA, wireless channel modeling, diversity, smart antennas, advanced signal processing and applications, communication network, ad hoc and mesh networks, and circuit and antenna design. He has published more than 40 papers.

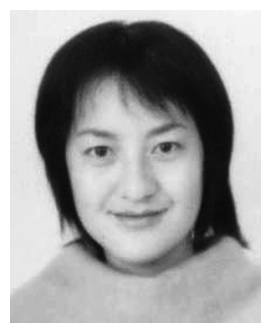

Xiujiang Huang was born in Sichuan, China, in 1977. She received the B.S. degree in applied electronics from Chengdu Institute of Information Engineering (CIIE) in 1999. She received the M.S. degree in electromagnetic field and microwave technology and the Ph.D. degree in optical engineering from University of Electronic Science and Technology of China (UESTC) in 2002 and 2005, respectively.

From March 2003 to April 2005, she was conducting research for her Ph.D. degree dissertation with the China Academic of Engineering Physics (CAEP), where she developed the ultrafast $\mathrm{Yb}^{3+}$-doped fiber ring laser and CW-LD Pumped Q-switched Nd:YVO4 Laser. Since July 2005, she has been a Research Scientist with the No. 23 Research Institute of China Electronics Technology Group Corporation. Her research interests include optic-electronic components, femtosecond and nonlinear optics, optical systems engineering, fiber-optic sensors, lasers, microwave circuit, and antenna design. She has published more than 30 papers.

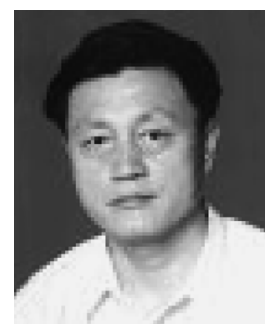

Zaiping Nie (SM'98) was born in Xi'an, China, in 1946. He received the B.S. degree in radio engineering and the M.S. degree in electromagnetic field and microwave technology from the Chengdu Institute of Radio Engineering (CIRE), Chengdu, China, in 1968 and 1981, respectively.

From 1987 to 1989 , he was a visiting scholar with the Electromagnetics Laboratory, University of Illinois at Urbana-Champaign. He is a Professor with the Department of Electromagnetic Engineering, University of Electronic Science and Technology of China (UESTC). He has published more than 300 papers. His research interests include computational electromagnetics, electromagnetic scattering and inverse scattering, waves and fields in inhomogeneous media, and smart antennas in mobile communications.

Prof. Nie is a fellow of the Chinese Institute of Electronics (CIE).

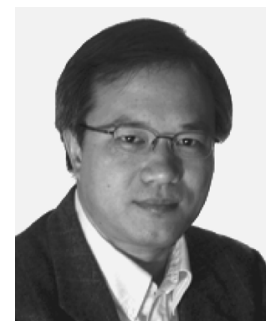

Yimin Zhang (SM'01) received the Ph.D. degree from the University of Tsukuba, Tsukuba, Japan, in 1988.

He joined the faculty of the Department of Radio Engineering, Southeast University, Nanjing, China, in 1988. He was a Technical Manager with the Communication Laboratory Japan, Kawasaki, from 1995 to 1997, and was a Visiting Researcher with ATR Adaptive Communications Research Laboratories, Kyoto, Japan, from 1997 to 1998. Since 1998, he has been with Villanova University, Villanova, PA, where he is currently a Research Associate Professor with the Center for Advanced Communications. His research interests include statistical signal and array processing for communications and radar applications, including space-time adaptive processing, wireless networks, MIMO systems, cooperative diversity, blind signal processing, digital mobile communications, time-frequency analysis, and source localization and tracking.

Dr. Zhang serves as an Associate Editor for IEEE SignAL PROCESSING LETTERS. 